Ann. Génét. Sél. anim., I977, 9 (2), I8I-20I.

\title{
Comparaison des races ovines Chios et Frisonne avec leurs croisements en Grèce continentale
}

\section{II. - Production laitière}

\author{
P. KALAISSAKIS, T. PAPADIMITRIOU, J. C. FLAMANT $(*)$, \\ J. G. BOYAZOGLU (**) et N. ZERVAS (**) \\ École Nationale Supérieure Agronomique, Athènes (Grèce) \\ (*) Station d'Amélioration génétique des Animaux, \\ Centre de Recherches de Toulouse, I.N.R.A. \\ B.P. I2, 31320 Castanet-Tolosan (France).
}

(**) Faculté d'A gronomie et de Sylviculture, Université Aristote, Thessalonique (Grèce).

\section{Résumé}

La présente étude concerne l'analyse des performances laitières de brebis de races pures Chios et Frisonnes ou issues du croisement entre ces deux races (FI, 3/4 Frisonnes, $7 / 8$ Frisonnes). La production moyenne des brebis traites Chios est légèrement supérieure à celle des brebis Frisonnes (I6I kg vs I $48 \mathrm{~kg}$ ). Compte tenu des difficultés d'adaptation des brebis Frisonnes, les performances des brebis $\mathrm{FI}$ sont nettement supérieures ( $\mathrm{I} 84 \mathrm{~kg}$ ) mais la production des $3 / 4 \mathrm{Fri}$ sonnes tombe au niveau de celle des Frisonnes de race pure. La comparaison de ces résultats avec ceux obtenus avec la race $A$ wassi et la prise en compte de l'ensemble des critères de production (reproduction, lait, croissance des agneaux) permettent de préconiser la solution d'une amélioration génétique par sélection en race pure plutôt que par croisement avec la race Frisonne.

\section{1. - Introduction}

Dans une précédente publication (ZeRvas et al., I975), nous avons présenté les informations concernant les paramètres de viabilité et de reproduction dans les troupeaux de brebis Chios (Ch), Frisonnes (Fr) et métisses (Ch $\times \mathrm{Fr}$ ) de la Station Expérimentale de l'École Supérieure Agronomique d'Athènes (Votanikos-Attique). De ces observations effectuées pendant neuf années, il résulte une nette supériorité des brebis Chios vis-à-vis des Frisonnes, une bonne adaptation des femelles $\mathrm{F}_{\mathbf{1}}$ aux conditions du milieu sec et chaud de l'Attique, ainsi qu'une dégradation des performances de reproduction et de viabilité (mère et agneaux) au-delà dẹ la pre- 
mière génération de croisement. Ces observations confirment les informations disponibles dans d'autres pays, en Europe méridionale (RICORdEAU et Flamant, I969; a CASU, I97I), dans les Balkans (ToDorovski et al., I970) et au Moyen-Orient (SHIMSHONi et LAVI, I972) concernant la difficulté pour la brebis de la Frise Orientale de vivre et de reproduire normalement dans les conditions climatologiques et dans le contexte des méthodes d'élevage pratiquées dans le Bassin Méditerranéen (Flamant et Ricordeau, I969; Flamant, I974), alors que cette adaptation ne semble pas poser de problèmes dans des localisations proches de son milieu d'origine, par exemple en Tchécoslovaquie (Cumlivski, I976).

Toutefois, comme nous l'avons dit précédemment (ZERvas et al., I975), " la comparaison entreprise ici est en fait beaucoup plus intéressante par les qualités de la race locale qu'elle met en évidence, que par les difficultés d'adaptation des brebis Frisonnes en milieu méditerranéen ". Les éléments complémentaires concernent ici la production laitière des brebis auxquels nous ajouterons, au niveau de la discussion générale, les résultats obtenus par PAPAdopoulos et al. (I972) sur la croissance des agneaux dans le cadre de la même expérimentation.

\section{2. - Matériel animal, méthodes d'élevage et de contrôle}

Les caractéristiques principales du système d'élevage réalisé à Votanikos et les paramètres climatiques moyens permettant de situer le milieu dans lequel l'expérimentation a été réalisée, ont été présentés précédemment (ZERVAS et al., I975); il en est de même pour les types génétiques concernés. Nous apportons ici seulement les informations complémentaires génétiques relatives à l'évaluation des performances laitières des mères.

Durant la période considérée (I959-I967), 257 brebis au total ont été en production, réalisant ainsi près d'un millier de lactations. Compte tenu du nombre restreint de brebis croisées présentant $15 / \mathrm{I}^{\mathrm{e}}$ et plus de sang Frison ( $^{\mathrm{e}}$ génération de croisement et au-delà), nous n'avons retenu pour les comparaisons effectuées ici que les lactations de brebis de race pure (Chios et Frisonne) et les produits des croisements de $\mathrm{I}^{\mathrm{re}}, 2^{\mathrm{e}}$ et $3^{\mathrm{e}}$ générations entre ces deux races (respectivement $\mathrm{I} / 2$, $3 / 4$ et $7 / 8$ de sang Frison). Nous n'avons d'autre part retenu dans les tableaux que les mises-bas réalisées par les brebis âgées de I à 7 ans, et nous n'avons tenu compte que des lactations ayant une durée minimum "valable " de Ioo jours en première mise-bas et de II5 jours pour les mises-bas suivantes (voir effectifs du tabl. 2).

Retenons aussi que la confusion année-génotype-âge, classique dans ce genre d'étude et pouvant avoir une incidence si les conditions d'élevage changent beaucoup d'une année à l'autre (ce qui n'est pas le cas ici), existe peut-être dans le contexte de cette étude mais, si ceci est le cas, elle ne peut malheureusement être ni évitée, ni corrigée.

\section{Période d'allaitement}

La durée d'allaitement est toujours de 56 jours, ce qui correspond à la pratique traditionnelle en Grèce. Toutefois, dans nos conditions expérimentales, les agneaux sont séparés en permanence de leurs mères dès le quatrième jour, sauf pour les deux tétées journalières $(4 \mathrm{~h} 30 / 5 \mathrm{~h}$ et $\mathrm{I} 6 \mathrm{~h} 30 / \mathrm{I} / \mathrm{h})$. Dès le septième jour après la 
mise-bas et durant les sept semaines suivantes jusqu'au $56^{\mathrm{e}}$ jour, on estime une fois par semaine la production laitière journalière des mères par pesée des agneaux avant et après tétée, le matin et le soir (RICORDEAU et al., I960; BoyAzogLU, I963). Nous disposons donc de 8 jours de contrôle hebdomadaire. L'estimation de la production laitière durant l'ensemble de la période d'allaitement est réalisée en considérant que la production est identique durant toute la semaine précédant un contrôle.

Dès l'âge de 12 jours, du foin de luzerne de bonne qualité et un mélange composé de 30/100 de maïs, 45/100 de son, 23/10o de farine de lin et 2/100 de sels minéraux sont mis à la disposition des agneaux ad libitum.

\section{Période de traite}

Après le sevrage, le contrôle laitier se fait à des intervalles approximatifs de dix jours (les 5 , I5 et 25 de chaque mois) par la pesée de la production laitière traite. L'évaluation quantitative de la production totale de la période de traite est réalisée par extrapolation : le premier contrôle est multiplié par le nombre de jours le précédant plus les cinq jours suivants, le second contrôle représente la période des dix jours suivants, ainsi de suite...

Une détermination du taux butyreux est faite durant la période de traite, par une prise d'échantillon représentatif lors de chaque contrôle laitier.

\section{Variables analysées}

Sept paramètres principaux ont été retenus pour réaliser les comparaisons des lactations entre les différents génotypes :

\section{Période l'allaitement}

- estimation $(\mathrm{kg})$ de la production laitière (PA);

- production au contrôle maximum (kg) (CMax A);

\section{Période de traite}

- durée (jours) (DT);

- production (kg) (PT);

- estimation de la production journalière moyenne (kg) (PJM T);

- production au contrôle maximum (kg) (CMax T);

- taux butyreux (\%) (TBT).

L'estimation de la production laitière durant la période d'allaitement (PA) ajoutée à la production traite (PT) constitue donc une indication sur le potentiel laitier total d'une brebis durant toute la lactation (production totale). 


\section{3. 一 Résultats}

\section{I. - Période d'allaitement}

Les informations concernant les quantités de lait tété par les agneaux durant les journées de contrôle hebdomadaire de la période d'ailaitement nous permettent une première évaluation des aptitudes laitières des brebis des différents génotypes en rapport avec le type génétique des agneaux allaités.

Les résultats relatifs à cette période sont présentés dans le tabl. I.

Le principal facteur de variation de la production par brebis est le nombre d'agneaux allaités, le sexe de l'agneau n'ayant aucun effet significatif. Durant les 56 jours d'allaitement, la production moyenne des brebis allaitant deux agneaux est en effet supérieure de 34 à 42 p. Ioo à celle des brebis allaitant un seul agneau, et celle des brebis allaitant trois agneaux de 62 à 92 p. Ioo.

En revanche, le génotype des brebis n'introduit que des différences relativement peu importantes, surtout dans le cas de la production laitière totale durant la période d'allaitement pour les brebis avec un seul agneau. Il est intéressant de remarquer que le génotype de l'agneau détermine apparemment le niveau de production de la mère autant que le génotype de celle-ci. C'est ainsi que la production laitière des brebis Chios allaitant un agneau $\mathrm{F}_{1}$ Frison $\times$ Chios est toujours un peu plus élevée que celle des brebis Chios allaitant un agneau de même race et atteint un niveau comparable à celui des brebis Frisonnes ou croisées Frisonne $\times$ Chios des différents numéros de génération.

\section{2. - Période de traite}

Les performances moyennes par génotype et âge de la mise-bas (tabl. 2) appellent les observations suivantes :

- la quantité moyenne de lait trait des brebis Chios est supérieure d'environ $8 \mathrm{p}$. Ioo (I6r kg contre I48) à celle des Frisonnes, ce qui est à rapprocher de la durée moyenne de lactation également inférieure pour les Frisonnes (I53 j contre I72). La caractéristique la plus intéressante est toutefois la grande stabilité de production laitière des Chios adultes ( 3 ans et plus) qui produisent plus de $170 \mathrm{~kg}$ de lait à la traite (même au-delà de 7 ans d'âge), tandis que la production des Frisonnes diminue dès l'âge de 4 ans avec des lactations relativement courtes. Ceci est confirmé par les estimations de la production journalière moyenne. Rappelons toutefois que la brebis Frisonne est réputée arriver à sa production maximum dès l'âge de trois ans.

On observe par ailleurs des coefficients de variation plus élevés pour les paramètres de production des Frisonnes que pour les Chios.

- Les résultats relatifs à la production au contrôle maximum (CMax T) ne traduisent pas la même évolution des performances d'une lactation à l'autre que celle qui a été observée pour la production totale. En effet, les lactations des brebis Frisonnes atteignent un niveau maximum plus élevé que celles des Chios à tous âges. La diminution sensible des performances totales des brebis Frisonnes au-delà de 3 ans se manifeste donc de préférence sur la deuxième moitié de la lactation (lactation écourtée accidentellement ou moins bonne persistance). Ceci est 


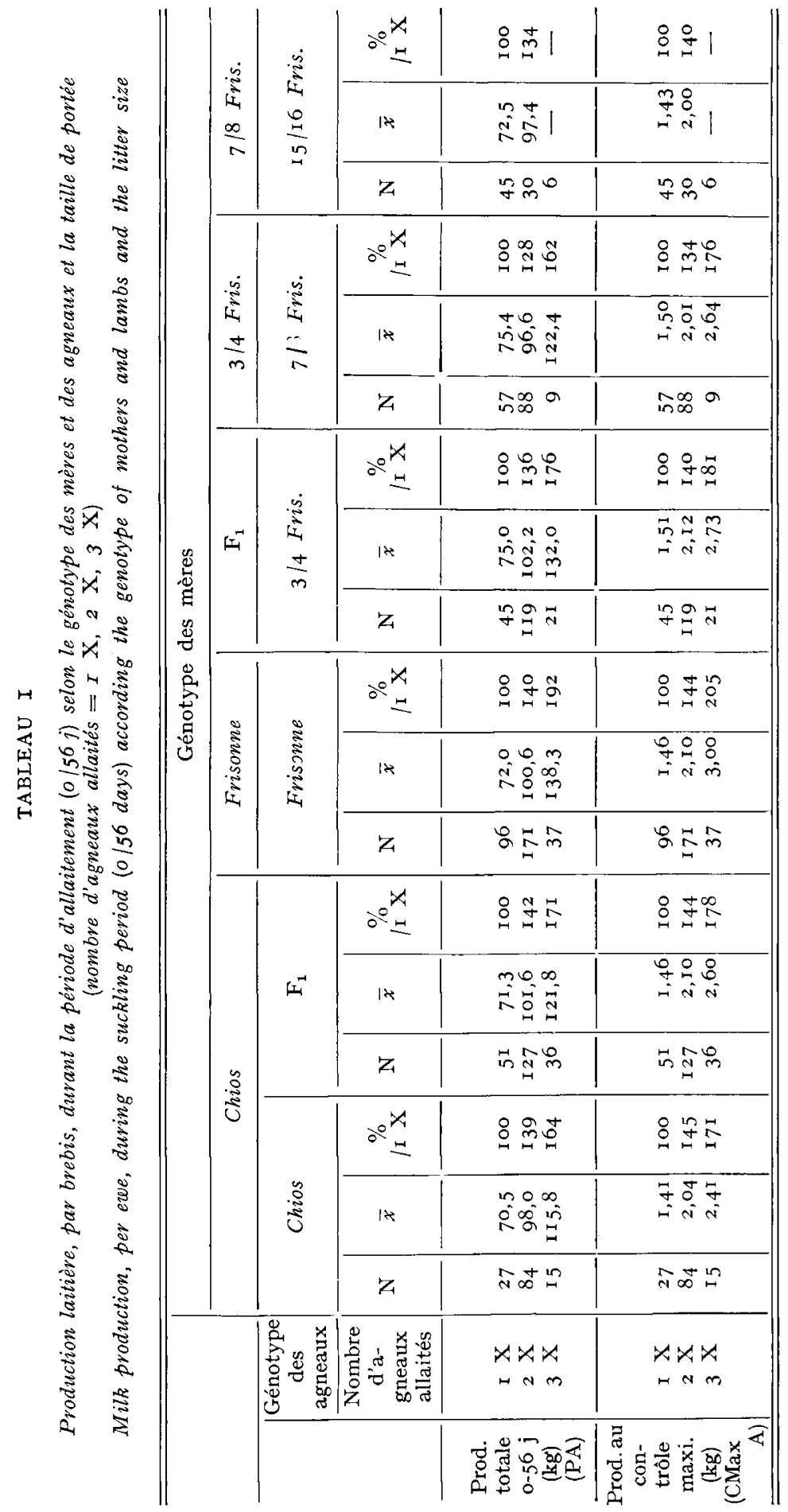




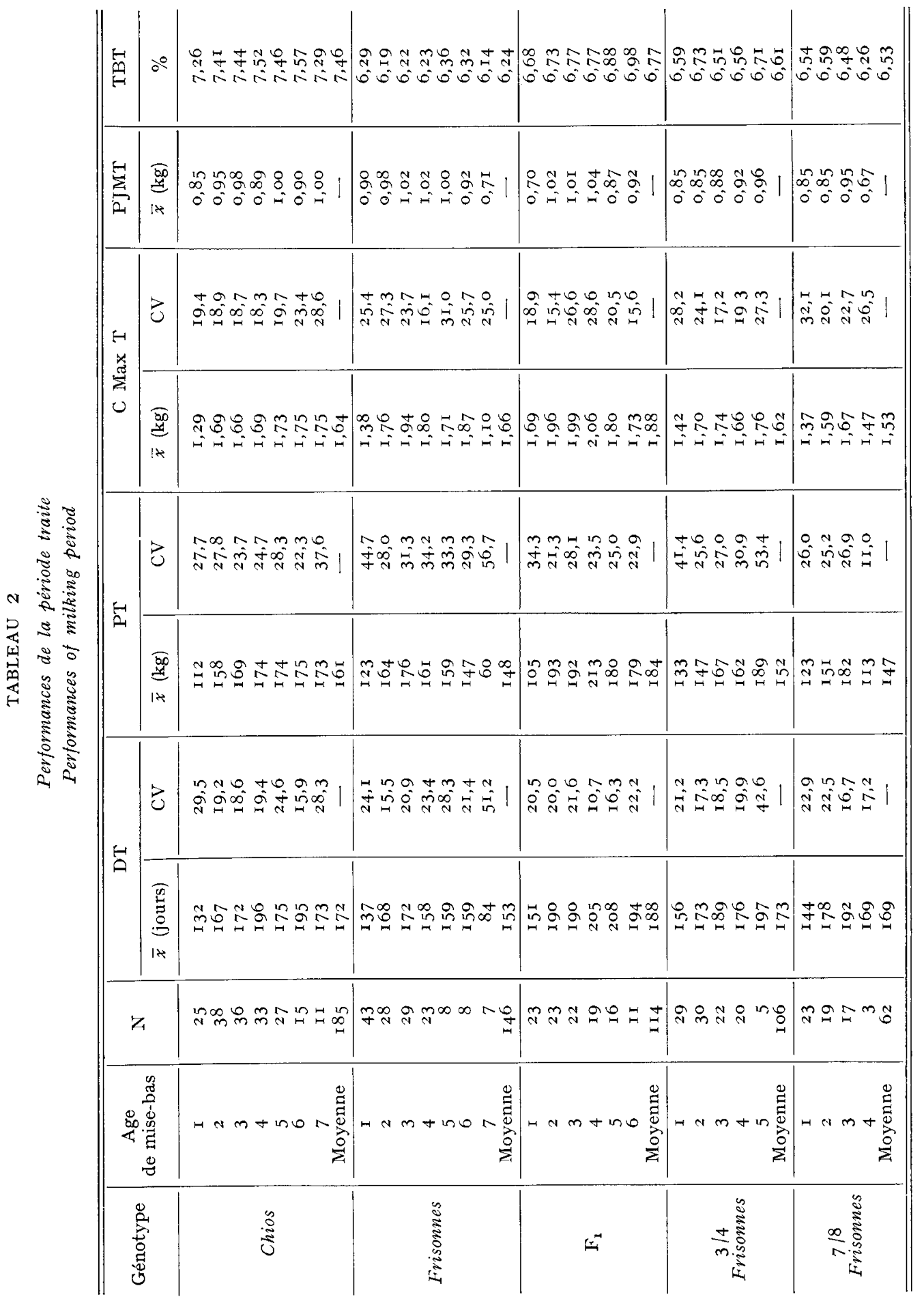


à mettre en rapport avec le fait que la comparaison a été réalisée en éliminant, dès le départ, un nombre de lactations " non valables » très supérieur pour la population Frisonne vis-à-vis de la population Chios, ceci à cause de lactations tronquées, d'accidents sanitaires, etc. Par rapport au nombre de mises-bas normales, le pourcentage de lactations retenu pour cette étude est de $96 \mathrm{p}$. Ioo pour les Chios et les 3/4, $93 \mathrm{p}$. Ioo pour les $\mathrm{F}_{1}$, et seulement $77 \mathrm{p}$. Ioo pour les Frisonnes pures et 85 p. IOO pour les $7 / 8$.

- En ce qui concerne la richesse du lait (TBT) exprimée en taux de matières grasses, nous trouvons dans tous les cas une très nette supériorité des Chios par rapport aux Frisonnes, les croisées étant intermédiaires.

- Les données de production révélent d'autre part la supériorité des métisses $\mathrm{F}_{1}$ par rapport aux deux races parentales à tous les âges (I $84 \mathrm{~kg}$ contre I6I et I48, respectivement pour la Chios et la Frisonne en ce qui concerne la quantité de lait produit à la traite). Cet avantage des $\mathrm{F}_{1}$ ne se manifeste plus, par rapport aux Chios purs, au-delà de $50 \mathrm{p}$. Ioo de sang Frison: les $3 / 4$ Frisonnes adultes produisant, à durée de traite égale (I7O-I75 j), 5-Io p. Ioo de lait en moins que leurs contemporaines de race Chios pure. La production des $7 / 8$ est pratiquement égale et même inférieure à celle des Frisonnes pures.

\section{3. - Courbes de lactations}

L'analyse des courbes de lactation n'est réalisée que sur les brebis adultes âgées de 3 ans et plus et ayant eu au moins Igo jours de traite après la période d'allaitement. Les courbes représentées dans la figure I concernent l'ensemble de la lactation de ces brebis sur une durée de 246 jours pour les quantités de lait et, dans la fig. 2, l'évolution des taux butyreux durant la période de traite.

Il est intéressant de noter que le contrôle maximum moyen de la lactation au cours de la phase d'allaitement (allaitement de 2 agneaux) est relativement tardif et se situe au cours de la $4^{\mathrm{e}}$ semaine de lactation pour les Frisonnes et au cours de la $6^{\mathbf{e}}$ semaine pour les autres génotypes (Chios, $\mathrm{F}_{\mathbf{1}}$ et $3 / 4$ Frisonnes).

Après un début de lactation se situant à peu près au même niveau moyen pour les Chios et les Frisonnes, on observe une moins bonne persistance pour ces dernières dont la courbe moyenne se situe toujours en dessous de celle des Chios. En revanche, la production plus élevée observée pour les $\mathrm{F}_{1}$ durant l'allaitement se maintient ensuite durant la période de traite. L'évolution de la lactation des brebis $3 / 4$ Frisonnes est très comparable à celle des Frisonnes pures.

Les brebis Chios se caractérisent aussi par une certaine constance des taux butyreux en cours de lactation : on n'observe pratiquement pas de phase d'enrichissement pour l'échantillon des brebis concernées. En revanche, la courbe des Frisonnes débute à un niveau plus bas que celle des Chios $(6,0 \%$ environ contre $7,3 \%)$ ou des croisées $\left(6,6 \%\right.$ pour les $\mathrm{F}_{1}, 6,4 \%$ pour les $\left.3 / 4\right)$ pour terminer pratiquement au même niveau $(7,4 \%)$ sans toutefois rattrapper totalement les Chios (7,7\%). Globalement, le lait des brebis Frisonnes et des croisées est donc moins riche que celui des brebis Chios.

\section{4. - Saison de mise-bas}

La saison de mise-bas, plus précoce pour les Chios que pour les Frisonnes (ZERVAS et al., I975) a une importante répercussion sur la production laitière traite : les agnelages tardifs de tous les génotypes (surtout au-delà de janvier) sont géné- 


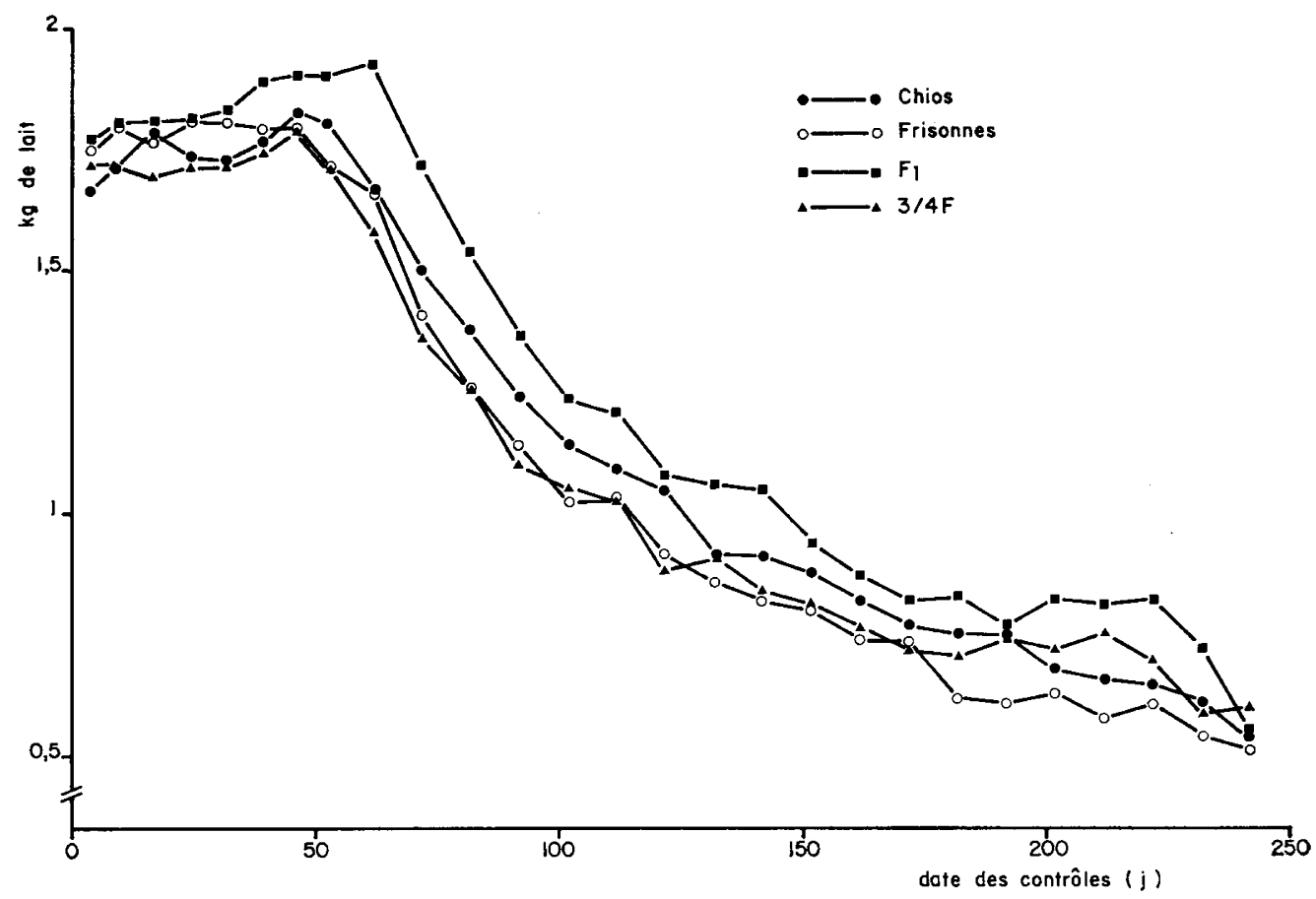

FIG. I. - Courbes de lactation (brebis adultes). Lactation curves (adult ewes).

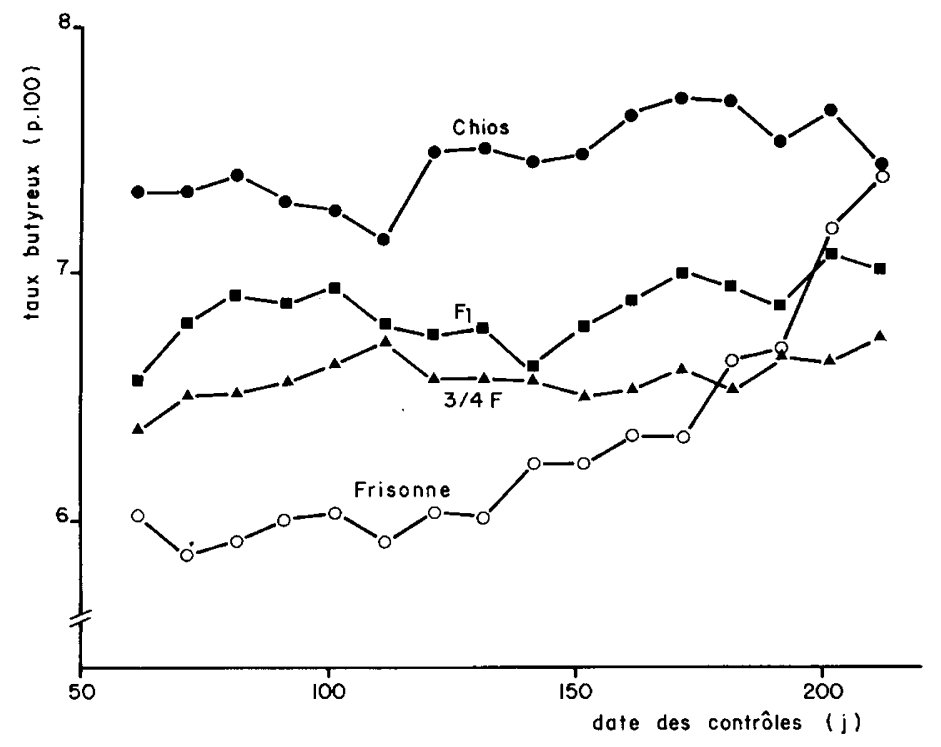

FIG. 2. - Taux butyreux pendant la période de traite. Fat percentage during milking period. 


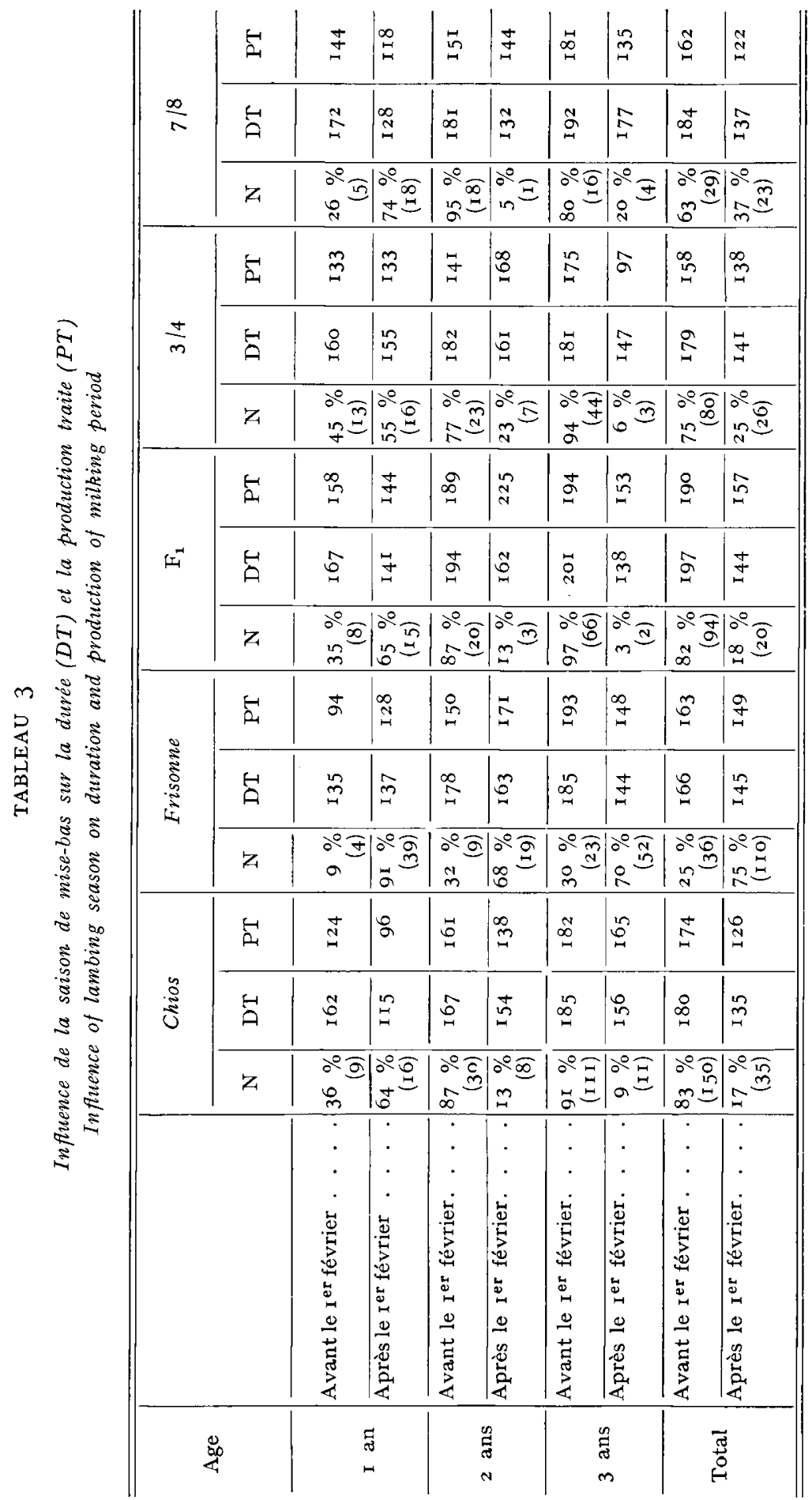


ralement suivis d'une lactation plus ou moins raccourcie avec des productions laitières en moyenne moins importantes à la traite (tableau 3). C'est ainsi que les femelles de race Chios (toutes lactations) qui ont mis-bas avant le I ${ }^{\text {er }}$ février représentent 83 p. Ioo de la population Chios étudiée, avec une durée moyenne de lactation de 180 jours et une production traite de I $74 \mathrm{~kg}$; les I7 p. Ioo qui ont mis-bas

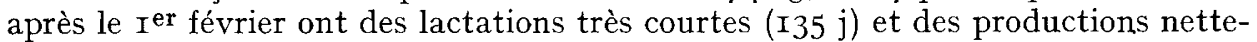
ment inférieures (I $26 \mathrm{~kg}$ ). Pour les Frisonnes, nous retrouvons le même phénomène avec toutefois $75 \mathrm{p}$. roo de lactations tardives ( $145 \mathrm{j}$ et I49 kg) contre $25 \mathrm{p}$. IOO de lactations précoces ( $166 \mathrm{j}$ et $\mathrm{I} 63 \mathrm{~kg}$ ).

Dans ces conditions, il est intéressant de comparer les performances des brebis des deux races pures, Chios et Frisonne en tentant d'éliminer l'effet du mois de mise-bas. C'est ce que nous avons fait en considérant uniquement les lactations réalisées après une mise-bas de janvier (tabl. 4), période au-delà de laquelle il peut $\mathrm{y}$ avoir distorsion entre les besoins de production et l'utilisation des ressources naturelles dans les conditions d'élevage de la Grèce. On observe alors que les durées moyennes de traite et les niveaux moyens de production pour les deux races considérées sont pratiquement identiques : I6o $\mathrm{kg}$ de lait pour 162 jours de traite et $159 \mathrm{~kg}$ de lait pour $\mathbf{5} 57$ jours de traite respectivement pour les brebis Chios et Frisonnes de tous âges.

\section{TABLEAU 4}

Performances moyennes des brebis Chios et Frisonnes ayant eu un agnelage au mois de janvier Mean performances of Chios and Friesian ewes having lambed in January

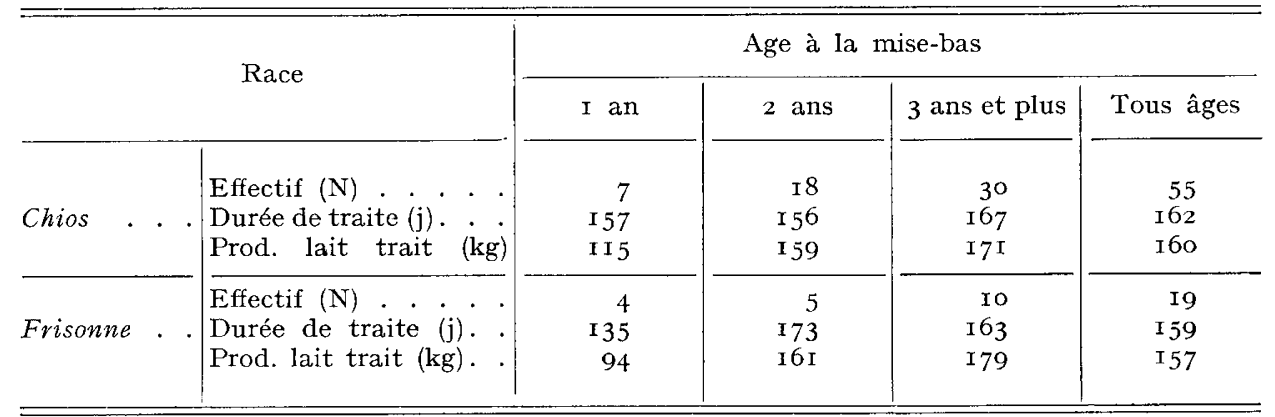

\section{TABLEAU 5}

Corrélations phénotypiques intra génotype $\left(\mathrm{r}_{x / y}\right)$ et régression $\mathrm{b}(y / x)$ entre la durée de traite $(\mathrm{x})$ et la production laitière à la traite (y)

Intragenotype Phenotypic correlation ( $\mathrm{r}$ ) and regression (b) between milking duration ( $\mathrm{x}$ ) and milk production during the milking (y)

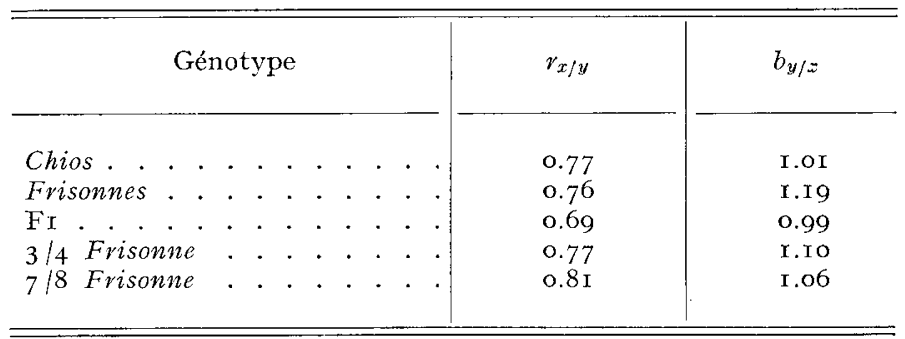


L'importance d'une mise-bas précoce sur la production traite est d'ailleurs confirmée par des corrélations $(r)$ élevées $(0,7-0,8)$ et des régressions $(b)$ de l'ordre de $I, O-I, 2$ calculées à titre d'information entre la durée de traite $(x)$ et la production traite $(y)$, pour chaque génotype sur les lactations de tous âges (tabl. 5).

\section{5. - Influence de l'année de production}

Le fait le plus important qui ressort de l'observation de l'évolution de la production traite d'une année de production à l'autre est la diminution progressive de la production des brebis Frisonnes, en valeur absolue et par rapport aux brebis Chios (fig. 3).
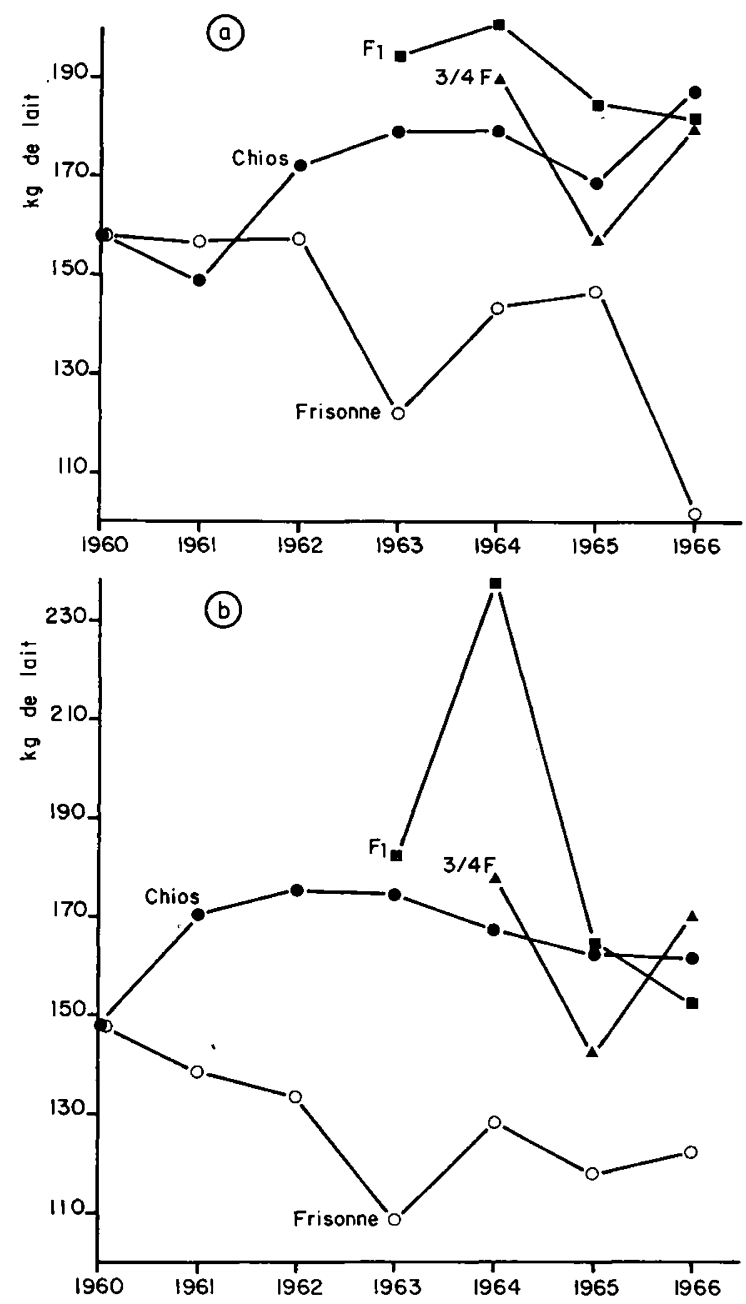

FIG. 3. - Evolution de la production laitière dans le temps.

a) brebis adultes; $b$ ) toutes les brebis.

Evolution of milk production with time.

a) adult ewes; $b$ ) all the ewes. 
Même si l'on prend en considération les seules lactations adultes (fig. $3 a$ ), l'on peut remarquer que, durant les trois premières campagnes, la production traite des brebis Frisonnes se maintient à peu près constante et à un niveau moyen égal à celui des Chios, alors que les années suivantes correspondent à la fois à une nette amélioration de la production des Chios et à une importante diminution chez les Frisonnes.

\section{4. - Discussion}

4.I. - Les travaux dont nous avons rapporté ici les résultats permettent d'abord de mieux préciser le potentiel de production laitière des brebis de race Chios.

Pour la période de traite, les performances moyennes obtenues à Votanikos durant les neuf années en question (I6I kg en I72 j de traite) sont quelque peu inférieures à celles observées par ZERVAs (I965) dans l'île de Chios — berceau de la race - sur 4979 lactations contrôlées (I74 kg). Mais l'amélioration des conditions d'élevage et une certaine sélection permettent d'atteindre des lactations adultes de l'ordre de $\mathrm{I} 8 \mathrm{o} \mathrm{kg}$ de traite au cours des dernières années. En ce qui concerne les meilleures lactations enregistrées dans la race, rappelons que ZERVAS (I965) obtient I6,8 p. Ioo de lactations supérieures à $240 \mathrm{~kg}$ de lait trait avec quelques brebis atteignant 350-50o kg. En Turquie, Sonmez et Wassmuth (1964) obtiennent une moyenne dépassant les $200 \mathrm{~kg}$ traits dans leur troupeau expérimental de l'Université d'Ege à Smyrne, des productions de l'ordre de $350-360 \mathrm{~kg}$ ayant été enregistrées pour quelques brebis (SoNMEz, I962 obtient 2 Io kg traits et $207 \mathrm{j}$ de traite en moyenne pour un "échantillon type " de $\mathrm{I} 2$ brebis). Ces résultats situent incontestablement les brebis Chios parmi les plus laitières de l'espèce ovine (BOYAZOGLU, I963), qualité qui vient donc s'ajouter à sa prolificité remarquable (ZERVAs et al., I975).

Pour la période d'allaitement, le niveau des performances est évidemment dépendant du nombre d'agneaux sous la mère. La particularité est ici que les naissances multiples sont la règle. Dans le cas relativement classique de deux agneaux allaités, l'accroissement du niveau de production laitière, de l'ordre de 30 à $40 \mathrm{p}$. Ioo est comparable à celui communément admis par la plupart des auteurs ayant travaillé sur d'autres races prolifiques (RICORDEAU et DENAMUR, I962; GOOT, I972). Les résultats concernant les portées triples sont par contre beaucoup plus rares. Flamant et LABUSSIÈRE, (I972) observent que la production des brebis Romanov allaitant trois agneaux est 62 p. Ioo supérieure à celle des brebis allaitant un seul agneau sur trois nés, durant le premier mois de lactation; nous obtenons une valeur légèrement supérieure pour les brebis Chios (64 et $7 \mathrm{I} \%$ ). Remarquons que cet accroissement est plus élevé lorsque les agneaux sont croisés Frisons. Cette observation peut être mise en rapport avec le potentiel de croissance plus élevé des agneaux Frisons (race pure ou croisée) par rapport aux agneaux Chios de race pure (poids moyens des agneaux doubles à la naissance : $\mathrm{Fr}=4,27 \mathrm{~kg}, \mathrm{I} / 2 \mathrm{Fr}=4,44 \mathrm{~kg}$, $\mathrm{Ch}=4,04 \mathrm{~kg}$; croissance journalière moyenne jusqu'à 56 jours : Fr $=264 \mathrm{~g}$, $\mathrm{I} / 2 \mathrm{Fr}=27 \mathrm{I} \mathrm{g}, \mathrm{Ch}=227 \mathrm{~g})$. L'on peut aussi faire le rapprochement avec la légère différence de production observée entre brebis Chios allaitant les agneaux de race pure ou des agneaux croisés $\mathrm{F}_{1}$ Frison $\times$ Chios. Ces écarts seraient la manifestation d'une plus grande sollicitation de l'activité secrétoire des brebis par des agneaux dont les besoins sont plus importants et ceci d'autant plus que la taille de portée est élevée. 
Papadopoulos et al. (I972) ont en effet bien mis en évidence, dans le cadre de cette même expérience, les plus grandes possibilités de croissance de la race Frisonne, de l'ordre de $\mathrm{I}_{5}$ à $20 \mathrm{p}$. Ioo, et qui se traduit en $\mathrm{F}_{1}$ par des croissances supérieures ou égales à celles des agneaux de race pure Frisonne (tabl. 6). La croissance des agneaux $3 / 4$ Frisons étant plus élevée que celle des agneaux $\mathrm{F}_{1}$, il est possible également que la production laitière des brebis $F_{1}$ bénéficie d'une certaine stimulation et soit donc légèrement surestimée par rapport aux brebis Chios allaitant des agneaux de race pure.

\section{TABLEAU 6}

Poids aux différents âges et croissances moyennes des agneaux exprimés en pourcentage des performances des agneaux de race pure Frisonne (Fr) (PApadopoulos et al., 1972)

Weight at different ages and meen growth of lambs measured in performance percentage of pure breed Friesian (Fr) lambs (PAPAdopoulos et al., 1972)

\begin{tabular}{|c|c|c|c|c|c|}
\hline & Chios & $F_{I}$ & $3 / 4$ & $7 / 8$ & $15 / 16$ \\
\hline \multicolumn{6}{|l|}{ Poids : } \\
\hline $\begin{array}{l}\text { A la naissance } . . . \\
(\mathrm{Fr}=4,4 \mathrm{~kg})\end{array}$ & $9 \mathrm{I}, 3$ & IOI, 2 & $\mathrm{IO}_{4}, 8$ & 98,9 & 92,8 \\
\hline $\begin{array}{l}\text { A } 2 \text { semaines } . \cdots \\
(\mathrm{Fr}=7,7 \mathrm{~kg})\end{array}$ & 88,4 & I0I, 8 & I09,7 & IO $4, \mathrm{I}$ & 97,0 \\
\hline $\begin{array}{l}\text { A } 4 \text { semaines } \\
(\mathrm{Fr}=\mathrm{I}, \mathrm{o} \mathrm{kg})\end{array}$ & 89,3 & 103,7 & I 12,6 & 105,8 & IOI, I \\
\hline $\begin{array}{c}\text { A } 6 \text { semaines } . . \\
\left(\mathrm{Fr}=\mathrm{I}_{5}, \mathrm{I} \mathrm{kg}\right)\end{array}$ & $86, \mathrm{I}$ & 101 6 & I I I , 8 & 105,3 & 99,2 \\
\hline $\begin{array}{l}\text { A } 8 \text { semaines } \\
(F r=19,9 \mathrm{~kg})\end{array}$ & 83,8 & 98,5 & I I 0,2 & 105,2 & IOI, 4 \\
\hline \multicolumn{6}{|l|}{ Croissance journalière : } \\
\hline $\begin{array}{l}\mathrm{O}-4 \underset{\text { semaines }}{(\mathrm{Fr}=24 \mathrm{I}} \mathrm{g}) \\
.\end{array}$ & 87,3 & 105,8 & I $x_{5}, 6$ & IO9, I & I 06,0 \\
\hline $\begin{array}{l}0-6 \text { semaines } \\
\left(\mathrm{Fr}=25^{8} \mathrm{~g}\right)\end{array}$ & 83,0 & 102,5 & I I 3,4 & го6,6 & I02,7 \\
\hline $\begin{array}{l}\mathrm{c}-8 \text { semaines } \\
(\mathrm{Fr}=279 \mathrm{~g})\end{array}$ & 80,7 & 100,0 & I 10,4 & I $06, \mathrm{I}$ & I03,6 \\
\hline
\end{tabular}

De ces observations, il résulte que l'on peut évaluer le niveau global de production des brebis adultes en ajoutant à la production traite la production tétée par deux agneaux (ce qui correspond à la taille de portée "moyenne »). Cette évaluation est rassemblée dans le tableau 7 pour les 4 génotypes Chios, Frisonne, $F_{1}$ et $3 / 4$ en prenant pour base des performances des brebis adultes ( 3 ans et plus).

4.2. - La comparaison entre les deux races pures Chios et Frisonne doit être raisonnée selon deux unités de temps : le stade de lactation, l'année de production.

En ce qui concerne le stade de lactation, il apparaît qu'en début de lactation (phase d'allaitement et production au contrôle maximum durant la phase de traite), 
TABLEAU 7

Estimation du potentiel total de production laitière par génotype (bvebis adultes) Estimation of total milk production potentiality per genotype (adult ewes)

\begin{tabular}{|c|c|c|c|c|}
\hline Production Génotype & Chios & Frisonne & $\mathrm{F}_{\mathrm{I}}$ & $3 / 4$ \\
\hline Allaitement $(56 \mathrm{j})\left({ }^{*}\right) . . . . .$. & 98,2 & 99,9 & 102,5 & 96,5 \\
\hline 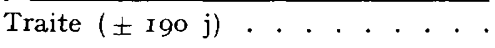 & I 79,9 & 165,0 & 201,5 & I 70,9 \\
\hline Production totale $( \pm 246 \mathrm{j}) .$. & $278, \mathrm{I}$ & 264,9 & 304,0 & 267,4 \\
\hline $\begin{array}{l}\text { Estimation du potentiel laitier adulte } \\
\text { (relatif) } . . \\
\end{array}$ & I $00,0 \%$ & $\pm 94,0 \%$ & $\pm 107,0 \%$ & $\pm 94,0 \%$ \\
\hline
\end{tabular}

(*) Esstimation de la quantité de lait tété par des agneaux doubles.

les deux races ont des performances très voisines. L'écart en faveur des Chios se manifeste essentiellement à partir du $3^{\mathrm{e}}$ mois de lactation : pourcentage inférieur d'accidents sanitaires susceptibles d'entraîner une interruption de la lactation (ZERVAS et al., I975), meilleure persistance de la production.

En ce qui concerne l'effet de l'année, l'évolution des conditions d'élevage au cours des neuf années de comparaison interfère certainement largement sur les performances enregistrées et complique notamment l'interprétation des résultats sur des effets tels que la saison de mise-bas. On a vu en effet dans la publication précédente concernant les critères de reproduction (ZERVAS et al., I975) que la date moyenne d'introduction du bélier dans le troupeau avait été avancée d'environ deux mois de I959 à I967. Cette modification a surtout avantagé les brebis Chios qui ont bien répondu par des mises-bas plus précoces permettant des performances plus élevées.

Les coefficients de variation plus élevés de la production traite des Frisons comparés à ceux des Chios sont peut-être dus à une plus grande variabilité des performances des Frisonnes liée à une très mauvaise adaptation de certaines brebis dans leur nouveau milieu d'élevage.

Par ailleurs, les difficultés d'adaptation des brebis Frisonnes ont entraîné la nécessité de conserver la quasi totalité des produits femelles en âge de reproduire alors que les bons résultats d'élevage des brebis Chios ont permis la réalisation d'une certaine sélection. Ces deux facteurs (meilleur désaisonnement et meilleure reproduction des brebis Chios par rapport aux brebis Frisonnes) expliquent sans nul doute que l'évolution des performances respectives des troupeaux de ces deux races se soit faite en sens contraire : amélioration des performances des brebis Chios, détérioration du niveau de productivité des brebis Frisonnes (fig. 3).

On observe ainsi que, partant d'un potentiel de production au moins aussi grand que celui des brebis Chios (sinon même supérieur), les performances globales des brebis Frisonnes sur l'ensemble des neuf années se révèlent inférieures par suite de l'influence de facteurs défavorables qui agissent :

- soit par effet direct sur la durée de la lactation et sur son niveau, notamment à partir du $3^{\mathrm{e}}$ mois après la mise-bas; 
- soit par effet indirect par l'intermédiaire des critères de reproduction qui déterminent le nombre de reproducteurs femelles disponibles et par conséquent la pression de sélection possible.

4.3. - Il est intéressant de situer les résultats de la comparaison ChiosFrisonne avec ceux concernant une autre grande race laitière du Bassin Méditerranéen, celle à queue grasse : l'Awassi. Plusieurs travaux sont actuellement disponibles dans la partie orientale de la Méditerranée (Israël, Liban, Chypre et Turquie) pour comparer la productivité globale de ces trois races dans les conditions d'élevage de chacun des pays considérés.

Les résultats des importations de reproducteurs de race Frisonne mâles et femelles en Israël et des expérimentations de croisements avec la race Awassi conduites pendant plusieurs années sont assez semblables à ceux des travaux conduits en Grèce : mauvaise adaptation des brebis Frisonnes, ce qui pénalise leurs performances laitières et les rend en moyenne inférieures à celles des brebis locales, légère supériorité des brebis $\mathrm{F}_{1}$ (et surtout $\mathrm{F}_{2}, \mathrm{~F}_{3}$ et $\mathrm{F}_{4}$ ) par rapport aux brebis locales (Goot, I966; Eyal et Goot, I968; Goot, I976 : tabl. 8). L'accroissement de production obtenu en $\mathrm{F}_{1}$ est cependant supérieur pour les brebis Frisonnes $\mathrm{X}$ Chios par rapport aux brebis Frisonnes $\times$ Awassi. Ceci peut être interprété de diverses façons : moindre adaptation des brebis Frisonnes ou effet d'hétérosis plus important en $F_{1}$ dans le cas de l'expérience grecque, ou niveau génétique un peu plus élevé pour les brebis $A$ wassi d'Israël. Les résultats obtenus à Chypre, comparant la production traite de brebis de races pures Awassi et Chios respectivement importées d'Israël et de Grèce, sont cohérents avec cette dernière hypothèse (Fox et al., I976). Les résultats du croisement entre ces deux races font suggérer par ailleurs l'existence d'un effet d'hétérosis important qu'il faudrait analyser de manière plus attentive (PAPAdopoulos, r966).

\section{TABLEAU 8}

Résultats préliminaires du croisement Awassi $\times$ Frison en Israël (Goor, 1976) (1) Preliminary results of Awassi $\times$ Friesian crossing in Israel (Goot, 1976)

\begin{tabular}{|c|c|c|c|c|c|c|c|c|}
\hline \multirow{2}{*}{\multicolumn{2}{|c|}{ Génotype }} & \multirow{2}{*}{$\begin{array}{l}\% \text { Relatif } \\
\text { de sang } \\
\text { Frison }\end{array}$} & \multicolumn{2}{|c|}{$\begin{array}{l}\text { Productions } \\
\text { laitières }(\mathrm{kg})\end{array}$} & \multicolumn{2}{|c|}{ Durée de lactation } & \multicolumn{2}{|c|}{$\begin{array}{c}\text { Début de lactation } \\
\text { (date) }\end{array}$} \\
\hline & & & $\bar{x}$ & D.S. & $\bar{x}$ & D.S. & $\bar{x}$ & D.S \\
\hline Awassi (A) . & . & 0 & 262 & $9 \mathrm{I}$ & $2 \mathrm{II}$ & 45 & $2 / I$ & 36 \\
\hline $3 / 4 \mathrm{~A} \cdot$ & . & 25 & $29 I$ & $6 \mathrm{I}$ & 218 & $4 \mathrm{I}$ & $23 / \mathrm{XII}$ & 13 \\
\hline I $1 / \mathrm{i} 6 \mathrm{~A}$. & . & 43,75 & 316 & I 20 & 229 & 49 & $\mathrm{I} 4 / \mathrm{XII}$ & 30 \\
\hline $\mathrm{F}_{\mathrm{I}} \cdot \dot{\mathrm{F}}_{\mathrm{I}} \times \dot{\left.\mathrm{FI}_{\mathrm{I}}\right)}$ & $\dot{.}$ & & 243 & 53 & 210 & 48 & $6 / I$ & 37 \\
\hline $\begin{array}{l}\mathrm{F}_{3}\left(\mathrm{~F}_{2} \times \mathrm{F}_{2}\right) \\
\mathrm{F}_{4}\left(\mathrm{~F}_{3} \times \mathrm{F}_{3}\right)\end{array}$ & . & $5^{\circ}$ & 283 & 96 & 222 & $5 \mathrm{I}$ & $21 / \mathrm{I}$ & 35 \\
\hline II / I $6 \mathrm{Fr}$ & . & 56,25 & 274 & 85 & $22 \mathrm{I}$ & 57 & $3 / 1$ & $4^{I}$ \\
\hline $5 / 8 \mathrm{Fr} .$. & 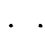 & 62,5 & 240 & 86 & 200 & 53 & $10 / 1$ & 40 \\
\hline $3 / 4 \mathrm{Fr}$. & . . & 75 & I 86 & 64 & I 86 & 52 & $2 \mathrm{I} / \mathrm{I}$ & 40 \\
\hline Frisonne $(\mathrm{Fr})$ & . . & IOO & I 82 & 80 & I 77 & 83 & $\mathrm{P} / \mathrm{I}$ & 60 \\
\hline
\end{tabular}

(I) Moyennes des femelles âgées de plus de deux ans, recueillies sur une période de I I ans (r958-59r969-7o) dans le troupeau de Newe Yaar. 
Les comparaisons effectuées au Liban (Fox et al., I976) et en Turquie (SoNMEZ et WASSMUTH, I964) avec des brebis Awassi locales sont au contraire à l'avantage net des brebis Chios. Pour le Liban, il s'agit toutefois d'une comparaison portant sur une quantité de lait estimée par traite durant la période d'allaitement et l'on peut penser que la taille moyenne de portée beaucoup plus élevée pour les brebis Chios a eu des répercussions positives sur la quantité de lait estimée à la traite après séparation des agneaux (BOyAzoglu, Ig63; EyAL et al., I973).

Pour confirmer ceci, remarquons que LAwlor et al. (I974) qui comparent la production laitière de brebis allaitant un seul agneau (avec traite après la tétée du soir) n'observent aucune différence entre deux échantillons de 33 brebis Awassi et Chios. Les résultats de LoucA et al. (I974) présentent un grand intérêt puisqu'ils permettent de mettre en rapport sur des brebis Awassi et Chios traites depuis 48 heures après la mise-bas, la consommation d'aliments, la variation de poids et la production laitière: avec une tendance à une meilleure production laitière et une moindre variation de poids vif pour une même consommation, on serait tenté ici d'attribuer à la brebis Chios une meilleure aptitude laitière. Des effectifs plus importants permettraient de mieux cerner ce problème.

Il faut également admettre que la sélection réalisée en Israël durant une longue période (FINCI, I957; FAI, I972) a amené le niveau génétique du rameau israëlien bien au-dessus de la moyenne des autres rameaux Awassi du Proche-Orient. Ce rameau devenant par ce fait une " autre race " moderne et améliorée, la production moyenne des troupeaux israëliens sous contrôle a passé de I3I kg en I938 à $367 \mathrm{~kg}$ en I973, la production maximum enregistrée en I938 était de $280 \mathrm{~kg}$ et celle de 1973 de I $233 \mathrm{~kg}$ (Israëli Breeders Assoc., I975).

4.4. - La figure 4, qui situe les niveaux respectifs des performances réalisées par les différents génotypes (Chios, $\mathrm{F}_{1}$, Frison $\times$ Chios, $3 / 4$ Frison, $7 / 8$ Frison, Frison pur) montre que la variation n'est pas la même selon les caractères considérés. La production laitière révèle une nette supériorité en $F_{1}$ puis une dégradation des performances au fur et à mesure que l'on se rapproche des Frisonnes pures. La même figure d'évolution se manifeste pour la durée de traite et la plupart des caractères de reproduction si l'on reprend les résultats de ZERVAS et al. (r975) : date moyenne de mise-bas, taux de fertilité, productivité numérique au sevrage des agneaux. En revanche, le taux butyreux se comporte comme un caractère strictement additif et les croissances moyennes des agneaux croisés ou de race pure Frisonne sont peu différentes entre elles mais sont supérieures à celles des Chios purs (PAPADOPOulos et al., I972).

D'un point de vue zootechnique, il est intéressant de juger les différents génotypes selon un certain nombre de caractères synthétiques $=$ quantité de matière grasse par lactation réalisée, quantité de matière grasse par brebis mise à la lutte, poids d'agneaux sevrés à 56 jours par brebis à la lutte. On observe alors que la moindre teneur du lait des $\mathrm{F}_{1}$ conduit à une quantité de matière grasse pratiquement semblable pour ces brebis à celle des Chios purs. La prise en compte des caractères de reproduction dans un bilan global conduit cependant à avantager les brebis $\mathbf{F}_{\mathbf{1}}$ à la fois pour la quantité de matière grasse produite et pour le poids d'agneaux sevrés par brebis à la lutte. Ce résultat est évidemment intéressant dans un élevage où tout le lait est destiné à être transformé en fromage et où un accroissement de la production de viande d'agneau est recherché (ZERVAS et al., I976).

Sur le plan génétique, cette étude met en évidence l'existence de deux types de caractères : les caractères de reproduction et de production laitière qui dépen- 

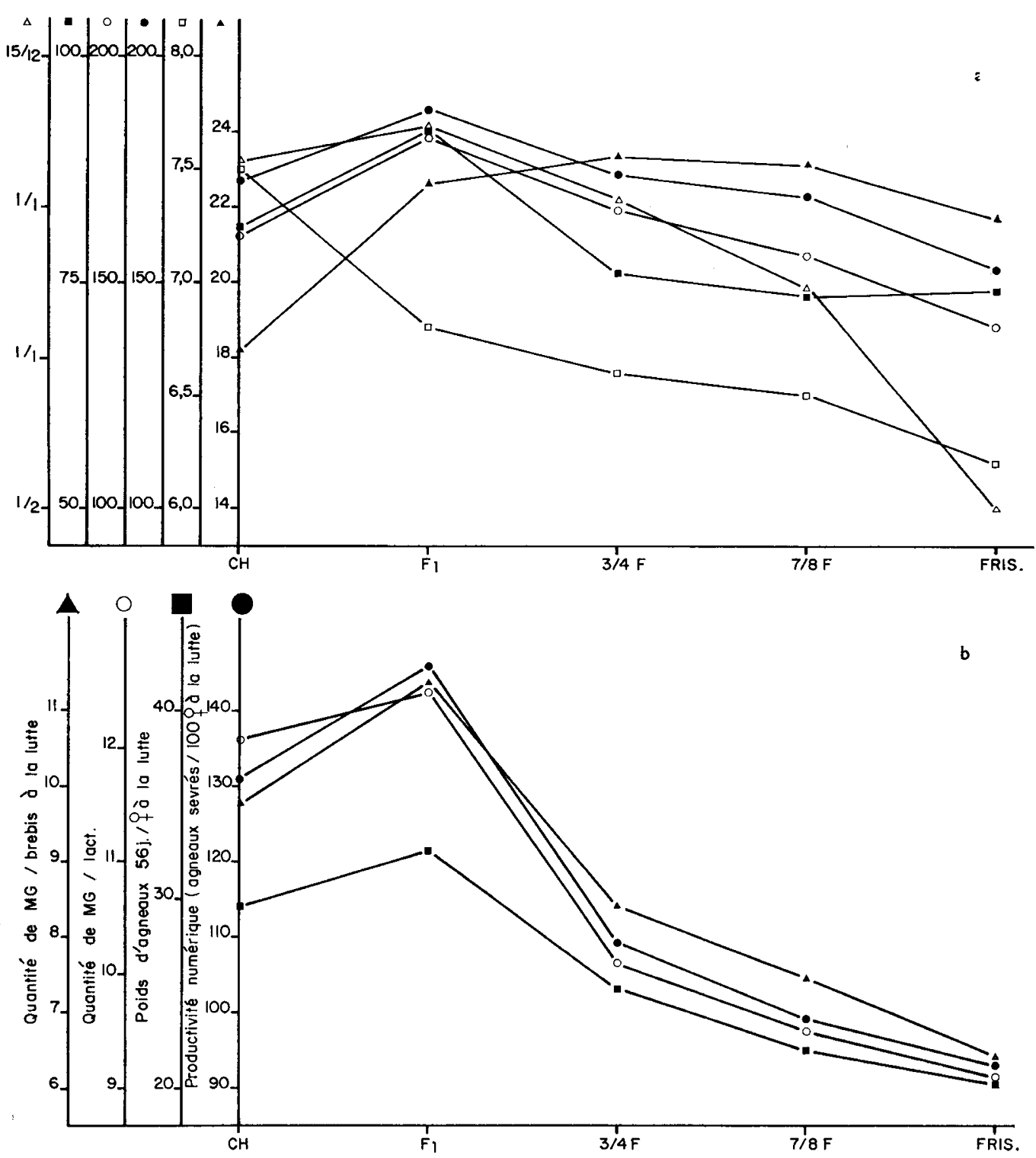

FrG. 4. - Niveau moyen des performances réalisées par les brebis des différents génotypes (tous âges et toutes années) pour les caractères de reproduction, la production laitière et la production d'agneaux.

a) Critères individuels (reproduction, lait, croissance).

b) Productivités globales (kilos de matière grasse ou d'agneaux pour Ioo brebis présentes à la lutte).

$\triangle$ date mise bas.

- Taux de fertilité (brebis saillies pour ıoo brebis mises en lutte).

- Production laitière traite $(\mathrm{kg})$

- Durée de traite (jours)

- taux butyreux (g. p. Ioo)

- Poids des agneaux à 56 jours. (kg.) 
dent directement ou indirectement de facteurs d'adaptation au milieu et pour lesquels on observe une supériorité des $F_{1}$, les caractères sans supériorité très apparente des $F_{1}$ tels que la teneur du lait en matière grasse et la vitesse de croissance des agneaux.

Cependant, comme l'avaient déjà souligné RicordeAu et Flamant (rg6gb), il est difficile dans le cas des croisements réalisés avec la race Frisonne de conclure à l'existence d'un effet d'hétérosis au sens strict du concept. Depuis, des expériences sur animaux de laboratoires ont pu montrer le rapport entre l'existence d'interactions génotypes - milieu et la manifestation d'un effet d'hétérosis (Orozco, I974). Dans le cas des caractères de croissance, une discussion en termes de vigueur hybride est en général délicate, compte tenu du fait du mélange des effets d'hétérosis direct et d'hétérosis maternel. Ceci est d'ailleurs exclu ici car une analyse plus complète est impossible puisque le schéma a été dirigé dans le sens d'un programme d'absorption.

4.5. - Ces travaux mettent une nouvelle fois en évidence les dangers d'un jugement de l'intérêt des reproducteurs Frisons sur la seule base des performances obtenues à l'issue d'un croisement de première génération (FLAMANT et RIcorDEAU, I969; RicordeAU et FLAMANT, I969b; FlamANT, I974) quel que soit le caractère parfois spectaculaire des résultats (Flamant et al., I975, Goot, I976; SonMEZ, I976; CASU, I977). Il est en effet indispensable d'envisager l'utilisation de ce résultat ponctuel dans une stratégie globale d'amélioration génétique. Les résultats décevants obtenus avec la race pure Frisonne ou au-delà d'un croisement de première génération, conduisent en effet à étudier l'intérêt respectif de plusieurs solutions : production de femelles $F_{1}$ à partir de brebis de race pure locale et non conservation pour la reproduction des produits de $F_{1}$, création d'une race synthétique (avec moins de $50 \%$ de sang Frison) à partir d'animaux croisés, sélection de la race locale pure. Compte tenu du niveau des performances atteint par les brebis Chios en production laitière tout comme de leur prolificité, on est tenté en première analyse de préconiser cette dernière solution. On ne peut naturellement rejeter l'intérêt éventuel d'exploiter la vigueur hybride qui se manifeste ici dans les $F_{1}$, toutefois ceci serait surtout intéressant dans un schéma simple pour la production de béliers $F_{1}$ produits par accouplements planifiés des meilleures brebis Chios par les meilleurs béliers Frisons et par insémination artificielle, tel que HickMAN (I976) l'a proposé. Ceci suppose toutefois l'existence de schémas de sélection bien développés, avec testage, pour les deux races paternelles, (la Chios en Grèce et la Frisonne en Allemagne), ce qui n'est pas le cas. De toute évidence l'étape prioritaire reste donc la mise en œuvre d'un programme national de sélection raisonnée de la brebis Chios en race pure.

\section{5. - Conclusion}

5.I. - La plupart des essais de croisements réalisés avec la race Frisonne dans les différents pays méditerranéens concerne des races locales dont le niveau de productivité est nettement inférieur à celui des brebis Frisonnes dans leur pays d'origine, et ceci pour tous les caractères quantitatifs : production laitière, reproduction, croissance, le seul avantage toujours perçu par les auteurs étant la meilleure rusticité des races locales. L'originalité du présent travail est de faire intervenir une race qui, avec un niveau de production laitière très proche, se situe 
parmi les races prolifiques et ne présente quele handicapéventuel d'un format moyen par rapport à un grand format. Compte tenu des conséquences d'une mauvaise adaptation de la race Frisonne, l'avantage global, dans les conditions climatiques de la Grèce passe à la brebis Chios. Mais il est évident aussi que, compte tenu des conditions d'élevage un peu particulières de la race Chios dans des milieux en moyenne plus favorables que pour la majorité des troupeaux grecques, qui utilisent des parcours, et de ses besoins importants durant les phases d'allaitement et de traite, la brebis Chios ne peut être préconisée dans toutes les localisations. Une telle étude de productivité globale devrait faire également intervenir la brebis Awassi en utilisant les paramètres disponibles.

5.2. - L'intérêt d'un inventaire génétique des races laitières et prolifiques méditerranéennes constitue certainement la deuxième leçon de ces travaux. La Méditerranée septentrionale et le Proche-Orient semblent en effet constituer un centre de différenciation génétique important pour l'espèce ovine, qu'il est indispensable d'inventorier et de protéger contre toute absorption intempestive par des races nord-européennes. Cet inventaire doit être réalisé en référence aux paramètres pouvant caractériser le milieu naturel et dans le cadre des systèmes d'élevage traditionnels.

Un tel inventaire devrait également être réalisé dans l'optique de la constitution de nouvelles combinaisons génétiques par croisements entre races " locales" reconnues intéressantes.

5.3. - L'intérêt de la mise en œuvre d'un schéma de sélection en race pure constitue en définitive la conclusion pratique essentielle de cet ensemble de résultats.

Dans l'immédiat et pour ce qui concerne plus spécialement la brebis Chios, l'on peut supposer qu'un effort minimum de sélection devrait permettre la réalisation de progrès notables à l'exemple de la race Awassi d'Israël dont les performances laitières ne semblent cependant que légèrement supérieures dans l'état actuel des choses.

Reçu pour publication en mai 1977.

\section{Remerciements}

Les auteurs tiennent à remercier MM. VISSAC, CASU, et FAI pour leurs suggestions quant à la rédaction finale de ce rapport.

\section{Summary}

A comparison of Chios and Friesian ewes with their various crossings in continental Greece

$$
\text { II. - Milk production }
$$

This study concerns the milk production of purebred Chios and East Friesian ewes and their crossbred progeny in an upgrading programme (FI, 3/4 and 7/8 Friesian) in Greece. It completes the previously published analysis of the reproduction performances of the same genotypes, by ZERVAS et al. (I975).

The milk-potential differences between genotypes are not significantly important at the 
beginning of the lactation (both the suckling period of 56 days and the first month of the milking period); the main differences appear after the third month of the lactation. During the milking period, the milk-yield level of the Chios ewes was constantly higher than that of the East Friesians $( \pm 8 \%)$.

A greater number of Friesian lactations had to be eliminated because of accidental interruptions (especially for sanitary reasons): 23 per cent vs. only 4 per cent for the Chios. Even after these eliminations the Friesian lactations were shorter in average length than those of the Chios (153 days vs. I 72 days).

The lower productivity of the Friesian ewes, established from the data collected during this 9-year period study, can be attributed to two main reasons. A direct effect of the environment on the level of production (influence of lambing-season and sensitivity to climatic and sanitary conditions) and an indirect effect of the environment causing reproduction difficulties which limit the possibility of selection.

The best productivity is obtained with the crossbred Fr ewes (Friesian $\times$ Chios) for all important parameters; be it for milk yield, reproduction ability or lamb production. The information collected for the $3 / 4$ and $7 / 8$ Fviesian crossbred females indicates that upgrading towards the purebred Friesian is not to be advocated.

A comparison of the very good productivity level of the Chios breed with that of the other high-yielding dairy breeds of this area, namely the Israeli Awassi and with the East Friesian and its crosses under mediterranean conditions, leads us to advise here the development of wellplanned purebred selection programmes rather than crossbreeding and upgrading schemes with high yielding but much less adaptable populations.

\section{Références bibliographiques}

Boyazoglu J. G., I963. Aspects quantitatifs de la production laitière des brebis. I. Mise au point bibliographique. Ann. Zootech., 12, 237-296.

CASU S., I97I. Essai de croisement entre les races ovines Sarde et Frisonne. C.R. Xe Congrìs intern. zootechn. (Versailles), Thème II, pp 4.

CASU S., 1977. Communication personnelle.

Cumlivski B., 1976. Reproduction and adaptation habilities of the East Friesian sheep in the C.S.R., 27th Annual Meeting, E.A.A.P., (Zurich), G 25 et S 33, pp 7 .

Eyal E., Folman Y., Morag H., I973. Lamb production in frequently lambing dairy sheep. World Rev. Anim. Prod., 9 (4), $64-69$.

Eyal E., Goor H., I968. Vital statistics and milk and laws production of Fi ewes (Awassi $\times$ East Frisian) under farm conditions (en Hébreu) Pamph. Nat. Univ. Inst. Agric. Rehovot, I 24, pp 26.

FAI 1., I972. Evolution de l'élevage ovin et du contrôle laitier des brebis en Israël. Symp. Milk Recording Practices Sheep Goats (Israël), I9-24 mars, pp 39.

Finci Mi., 1957. The improvement of the Awassi breed of sheep in Israël. Bull. Res. Counc. Israel, Vol. $6 B, \mathrm{pp}$ Iо6.

Flamant J. C., I974. Performances des brebis croisées en vue de la production laitière à la traite. ier Congrès Mond. Génét. Appl. Elev. (Madrid), 973-977.

Flamant J. C., Coste E., Fayot M. E., Ferrie H., i 975 . Amélioration génétique de la production laitière des brebis traites par l'utilisation de la race Frisonne. Journées de la Recherche Ovine et Caprine, I.N.R.A. ITOVIC, Paris 2-4 déc., Tome II, (Espèce Ovine), I73-180, SPEOC Paris.

Flamant J. C., Labussière J., i972. Premières observations sur les aptitudes laitières des brebis de race Romanov. Ann. Zootech., 21, 375-384.

Flamant J. C., Ricordeau G., 1969. Croisements entre les races ovines Préalpes du Sud et Frisonne. I. La brebis laitière de Frise Orientale. Élevage en race pure. Utilisation en croisements. Ann. Zootech., 18, 107-I 30.

Folman Y., Volcani R., Eyal E., 1962. Investigation of the accuracy of milk recording in A wassi flocks with proposal for improvements. Israel J. agric. Res., 12, I2I-1 30.

Fox C. W., Choueiri E., Chabaan R., 1976. The results of crossbreeding between Chios and the local fat-tail Awassi. 27th Annual Meeting, E.A.A.P., (Zurich), G 23 and S 3I, 9 p.

Goor H., I966. Studies on the native Awassi sheep and its crosses with the exotic East Friesian milk sheep (en Hébreu). Pamph. Nat. Univ. Inst. Agric. Rchovot, I15, pp 268. 
Goor H., I972. Milk yield and lactation of single and twin-rearing dairy ewes. Symp. Milk Record Pract. Sheep Goats (Israel), I9-24 March, pp 9.

Goox H., 1976. Scientific activities I971-r974. Institute of Animal Science, A.R.O. (Isvael), pp. 30.

Hickman C. G., I976. Hybrid breeding in cattle. Internordic Conference, Dairy cattle breeding in 80 s (Sweden), 9-Io December, pp 29.

ISRAELI SHEEP BREEDERS ASSOCIATION, 1975. The improved Israeli Awassi Sheep. Special pamphlet, pp 12.

Lawlor M. J., Louca A., Mavrogenis A., I974. The effect of the three suckling regimes on the lactation performance of $C y p r u s$ fat-tailed, Chios and $A$ wassi sheep and the growth rate of the lambs. Anim. Prod., 18, 293-300.

Louca A., Mavrogenis A., Lawlor M. J., 1974. Effect of plane of nutrition in late pregnancy on lamb birth weight and milk yield in early lactation of Chios and Awassi sheep. Anim. Prod., 19, 34I-350.

Orozco F., I974. Quantitative Genetics, Ier Congrès Mondial Génét. Appl. Elevage, Madrid, 7-IT Octobre, 1, 329-337.

Papadopoulos J. C., 1966. Republic of Cyprus, Annual Report of the Dept. Animal Husbandry for the year $I 965$, pp 48 .

Papadopoulos G., Kalaissakis P., Zervas N., Papadimitriou T., r972. Étude des races ovines Chios, Frisonne et leurs croisements en Attique II. Croissance des agneaux et conditions d'allaitement (en Grèce). Bull. Special du Ministère de l'A griculture de Grèce, pp. 2 I.

Ricordeau G., Boccard R., Denamur R., i96o. Mesure de la production laitière des brebis pendant la période d'allaitement. Ann. Zootech., 9, 28-120.

Ricordeau G., Denamur R., i962. Production Iaitière des brebis Préalpes du Sud pendant les phases d'allaitement, de sevrage et de traite. Ann. Zootech., 11, 5-38.

Rrcordeau G., Flamant J. C., i969a. Croisements entre les races ovines Préalpes du Sud et Frisonne. II. Reproduction, viabilité, croissance, conformation. Ann. Zootech., 18, I3II 49.

Ricordeau G., Flamant J. C., I $969 b$. Croisements entre les races ovines Préalpes $d u$ Sud et Frisonne. III. Performances laitières. Ann. Zootech., 18, I5I-I68.

Shrmshoni A., Lavi A., I972. Several veterinary and zootechnical aspects in the breeding of East Friesian sheep and their crosses. Symp. Milk Record. Pract. Sheep Goats (Israel), I9-24 March, pp 9 .

Sonmez R., I962. Breed characters and different productions in the Sakiz sheep (Chios Schafe) of Turkey, Z. Tierz. ZüchtBiol., 78, 28I-286.

Sonmez R., 1976. Crossbreeding experiments in native breeds of sheep in Turkey. World Rev. Anim. Prod., 12 (3), 83-86.

Sonmez R., Wassmuth R., r964. Untersuchungen über die Möglichkeiten der züchterischen Verbesserung der Milchleistung von Awassi-(Ivesi-), Sakiz-(Chios-), und Kivir-cikschafen in der Türkei. Züchtungskunde, 36, 23-30.

Todorovski N., Ristovski K., Dimitrovski D., Popovski K., Angel kov B., Kazic B., i97o. Model of a farm for the intensive breeding of dairy sheep with industrial methods (en Serbe). Veterinarski Glasnik, 24 (II), $855-858$.

ZERVAS N. P., I965. Contribution à l'étude phénotypique et génétique de la production laitière de la race Chios (en Grec). Thèse d'agrégation. Dept. Zootechnie, Ecole Supérieure Agronomique Athènes, pp $\mathbf{1} 20$.

Zervas N. P., Boyazoglu J. G., Kalaissakis P., Papadimitriou T., Flamant J. C., I975. Comparaison des races ovines Chios et Frisonne avec leurs croisements en Grèce continentale. I. Viabilité et reproduction. Ann. Génét. Sél. Anim., 7, 277-291.

Zervas N. P., Matsoukas J., Boyazoglu J. G., i976. La production de viande ovine et le croisement industriel en Grèce in Bull. tech. Dép. Génét. anim. (Inst. nat. Rech. agron., Fr.), $\mathrm{n}^{\circ} 25, \mathrm{I} 4-44$. Le croisement industriel ovin dans les pays méditerranéens. 